\title{
ORTHODONTIC-PROSTHETIC APPROACH IN THE TREATMENT OF COMPLEX CLINICAL CASES
}

\author{
Desislava Konstantinova, Hristina Arnautska. \\ Department of Prosthetic Dental Medicine and Orthodontics, Faculty of Dental \\ Medicine, Medical University - Varna, Bulgaria
}

\begin{abstract}
:
Modern dentistry demands an interdisciplinary approach in solving severe clinical cases. For a successful prosthetic treatment a number of prior manipulations are required. Rehabilitation of the oral cavity includes professional oral hygiene, repeated treatments of poorly filled root canals and removal of persistent teeth and roots. However, to achieve better conditions for prosthesis, special methods before the procedures are preferred. These methods include the more invasive periodontics and surgical techniques as well as the less invasive orthodontics techniques.
\end{abstract}

Key words: prosthetic dentistry; orthodontic; esthetics; hipodontia;

\section{INTRODUCTION:}

As a specialty prosthetics aims to restore the morphological, functional and aesthetic defects of the masticatory apparatus of the patient. $[3,4]$ Depending on the etiology and pathogenesis of the disease, the treating dentist has to draw up an appropriate treatment plan.[6] However, there are increasingly more patients with multiple dental problems which prevents immediate restoration of defects of the teeth rows. The preparation of the mouth for prosthesis includes rehabilitation and subsequent special pretreatment. A combined orthodontic-prosthetic treatment is widely used in modern dental practice. $[1,5]$ It involves creating a place for restoration of missing teeth, straightening teeth axes and correction of the Godon/Popov phenomenon in order to preserve the vitality of the bridge pillars. This approach allows for better conditions for aesthetic and functional prosthetic treatment in the long term. [4]

\section{CLINICAL CASE:}

The present paper describes the case of a nineteen-year old woman from Varna region. The patient sought help from a prosthetics specialist for aesthetic reasons - she did not like her smile and had impaired masticatory function and to a lesser extent had impaired speech function due to lack of more teeth in the upper jaw. The patient has suffered from Thalassemia Minor without any report of family history in relation to dental health.

After a clinical and X-ray screening the following diagnosis was given: Hypodontia in teeth 12, 14, 15, 45; Hypoplasia in tooth 22, persistent deciduous teeth 65 and 85 . A midline deviated to left was also established. As a result of extrusion (Godon phenomenon) and medial tilt around tooth 26, the diagnosis also confirmed: occlusion-articulation blockages, abnormal curve of Spee and lack of normal operation of Tilman's physiological strength due to spacing. Poor oral hygiene and presence of Gingivitis were also observed. (Fig. 1)
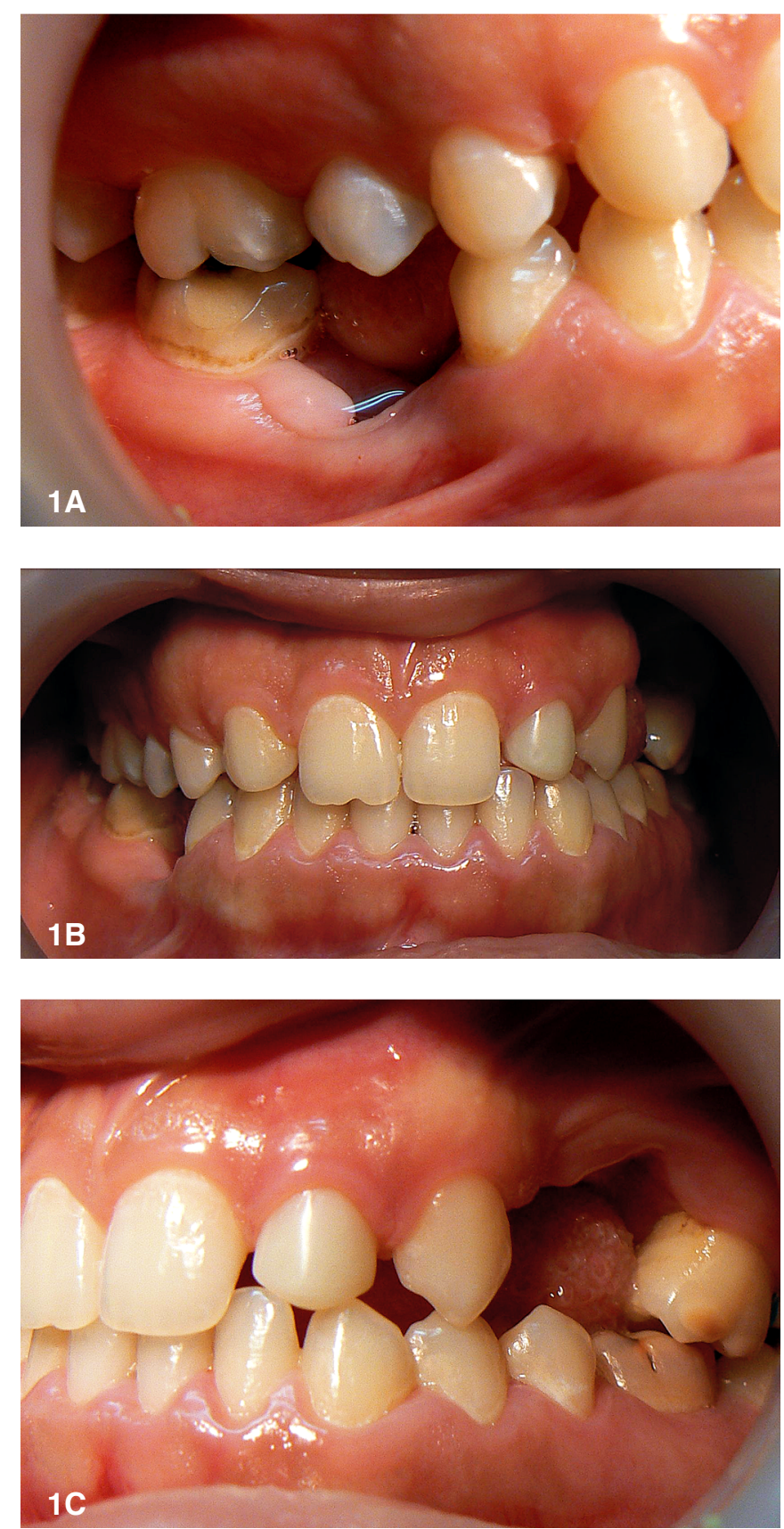

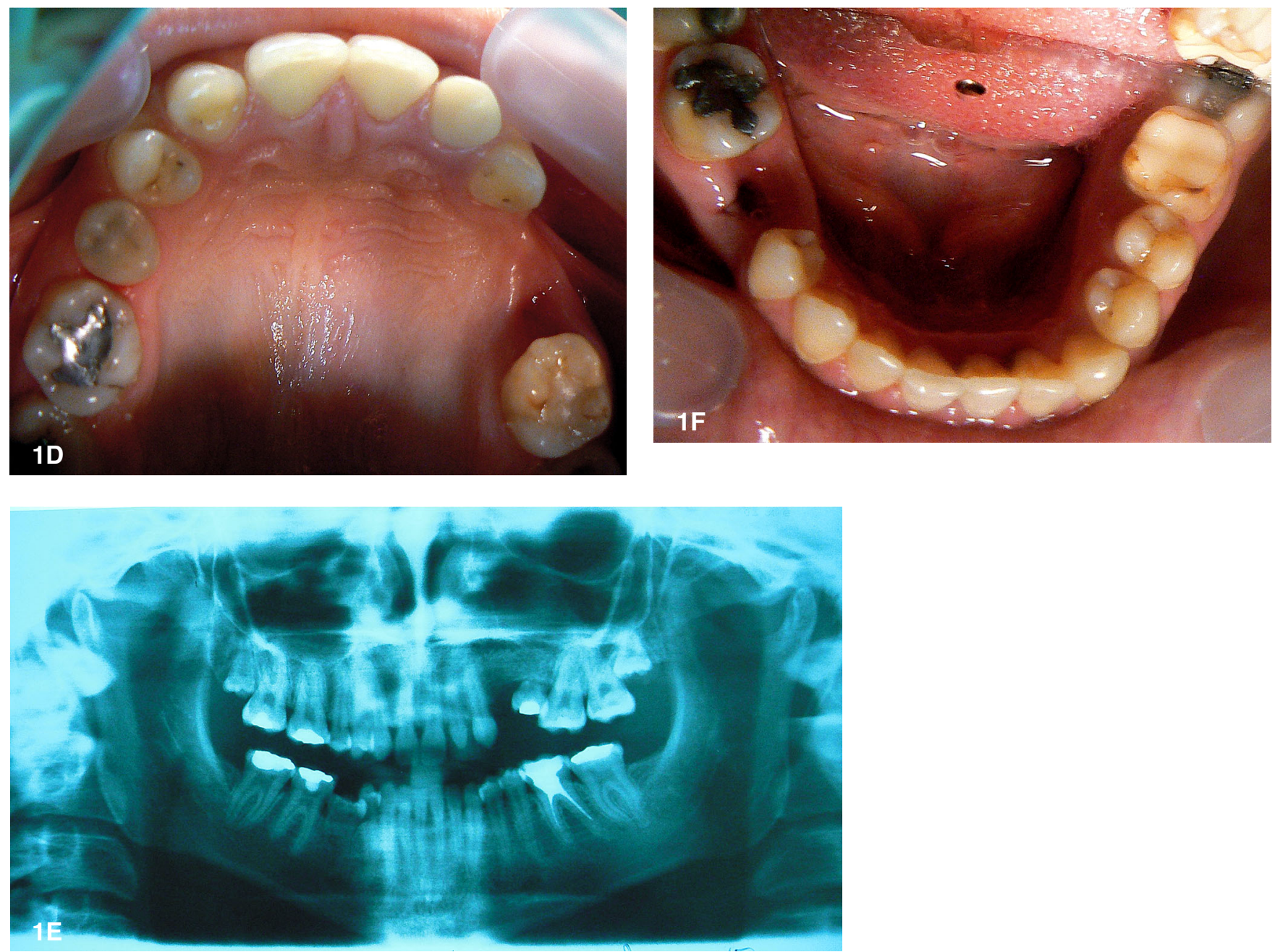

Fig. 1. Pretreatment intraoral photographs and panoramic radiograph

The therapeutic purpose for this patient is to achieve normal occlusal contacts, preparation of bridge pillars and correction of defects by placing a permanent structure. In order to create better conditions for prosthesis the following options for the preparation of the prosthetic area were offered:

1. Leveling of the occlusal plane and improved occlusal contacts by orthodontics methods, followed by Scaleing and Root planning and subsequent augmentation and placement of implants and single crow implants or metal-ceramic bridges on vital teeth.[2]

2. Leveling of the occlusal plane by methods of dental surgery and endodontics, again followed by periodontal and surgical preparation and placement of implants and single crow implants or metal-ceramic bridges on endodontically prepared bridge pillars.

The patient agreed to an orthodontic pretreatment, less invasive aiming to preserve the vitality of the remaining dentition. The next step was rehabilitation of the oral cavity and extraction of persistent teeth as well as periodontal preparation (curettage). Thus the suspected prognosis
(Prognosis dubia) turned into a hopeful prognosis (Prognosis bona). When given the choice of a prosthetic plan for financial reasons the patient refused implant treatment and chose bridging structures, i.e. bridge pillars.

The orthodontic treatment was conducted over the period of 15 months. MBT fixed appliance system with 022 slot was used. The orthodontic plan included medial movement of teeth 22 and 23 in order to close the existing spacing and achieve Class I around the left canines. For the purpose of normal occlusal contacts in the left molars, the medial tilt and overgrowing of tooth 26 were adjusted. Due to hypodontia in tooth 12 it was decided to medialise the canine in place of the lateral incisor and modeling its crown by enamel shaping where tooth 14 took the place of the right canine.

In the lower jaw teeth 44,43 and right central and lateral incisor were spaced to restore the midline and achieve Class I in the right canines. Also, the prosthetic area for tooth 45 was optimized. Likewise, correction of the curve of Spee was carried out. (Fig.2) 

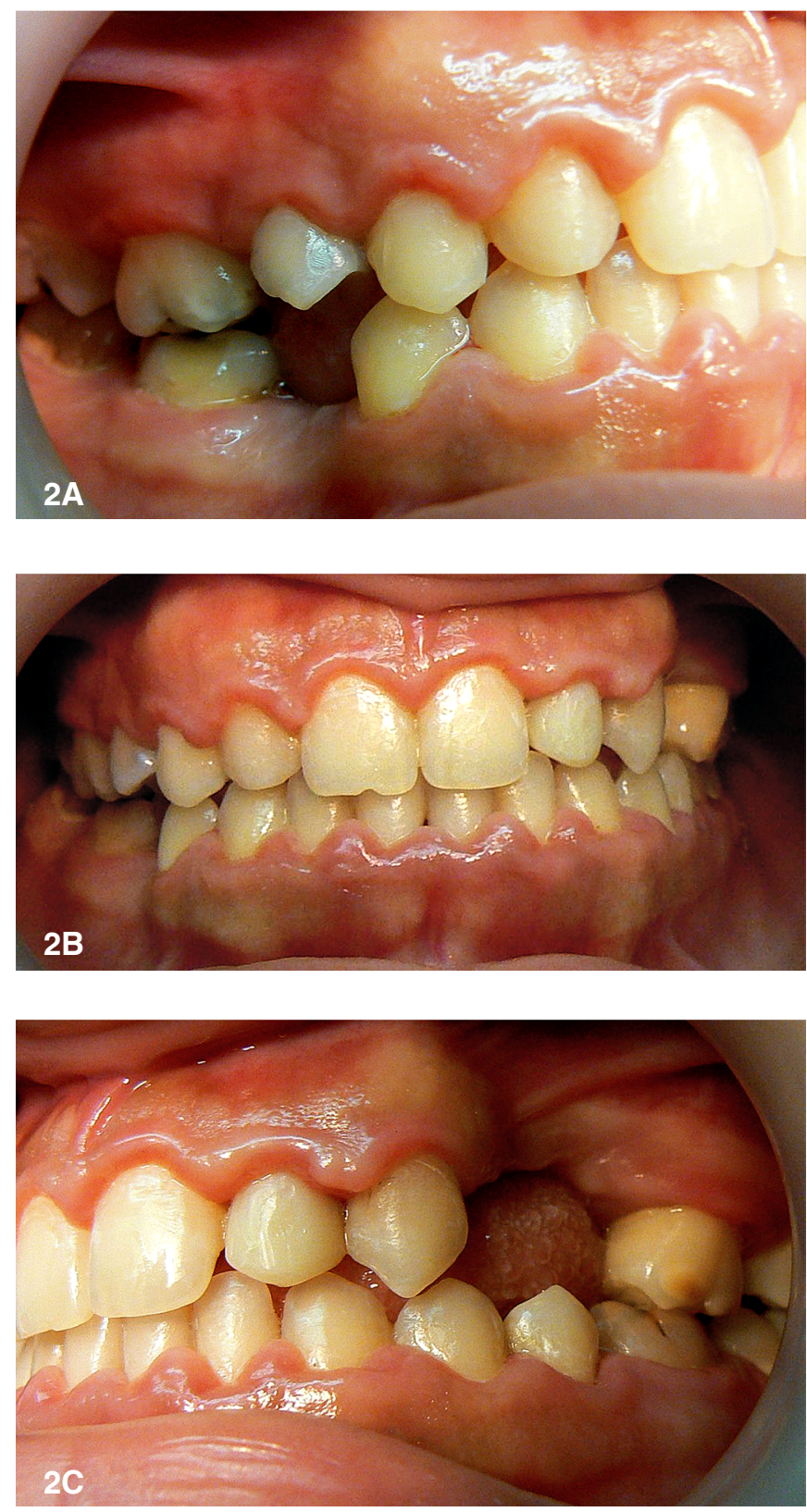

Fig. 2. Posttreatment intraoral photographs.

\section{CLINICAL RESULTS:}

Following the orthodontic treatment Class I was reached and medial-distal and vertical distance was modified for a prosthesis. The conditions for the physiological transmission of masticatory pressure onto bridge pillars and functional endurance of the prosthesis were improved.

The patient refused periodontal and implant treatment for financial considerations. That decision thus determined the choice for a permanent metal-ceramic structure transmitting the physiological masticatory pressure by means of tooth - periodontium - bone. Considering the optimal prosthetic area and the patient's requirements, the bridges were designed and constructed on their own supporting teeth around teeth $23,24,25,26,45$ and 46, which was done in view of the aesthetic and functional outcome.
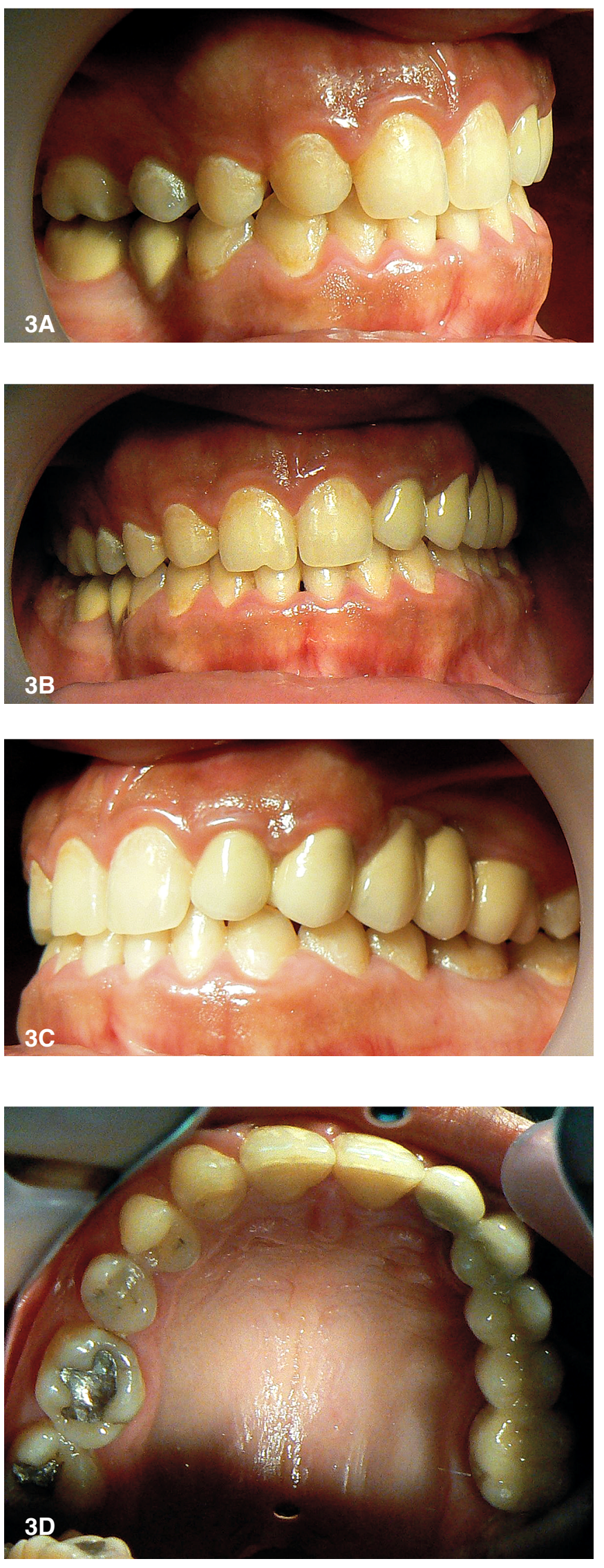


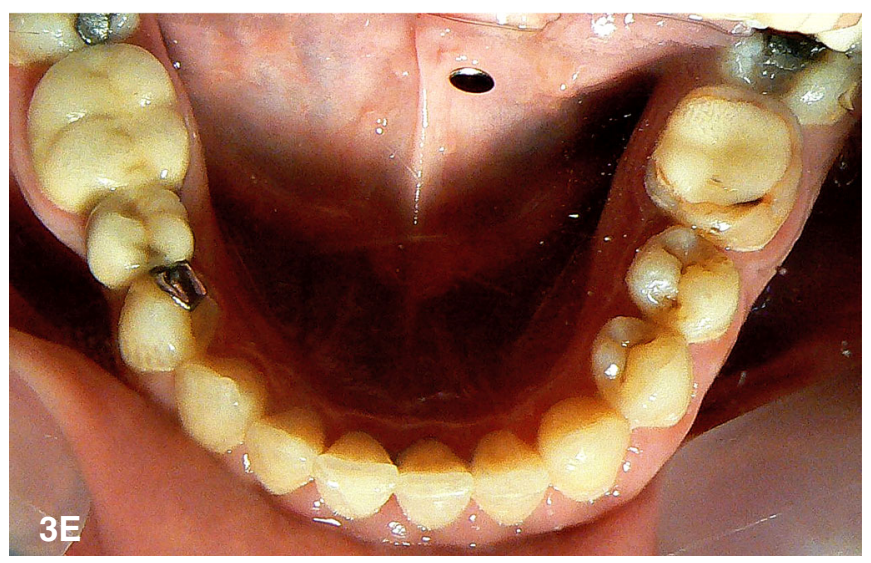

Fig. 3. Final results

From an aesthetic and functional point of view the results are good. The patient was satisfied, hoping for improved opportunities of social and professional development.

\section{DISCUSSIONS:}

The chances of success in the recovery treatment for patients with multiple problems of the masticatory apparatus have considerably increased with an orthodontic pretreatment preparation. [4, 5] The vitality of all teeth with bridge pillars is preserved thanks to prior intrusion and shaping of occlusal plane. Filing of hard dental tissues for facets and/or crowns (teeth 13,22) is avoided in the frontal area, also thanks to the orthodontic preparation. Good interdental contacts and physiological transmission of masticatory pressure were achieved, enabling more occlusal vertical forces to act along the axial surface of the bridge pillars. This is a prerequisite for the development of functional and aesthetic prosthesis.

\section{REFERENCES:}

1. Arslan SG, Tacir IH, Kama JD. Orthodontic and prosthetic rehabilitation of unilateral free-end edentulous space. Aust Dent J. 2006 Dec;51(4): 338-41. [PubMed] [CrossRef]

2. Georgiev T, Peev S, Papanchev G, Papancheva T, Djongova E. A clinical case of implant placement with guided bone regeneration, II-nd Scientific Congress of Bulgarian Dental So- ciety, Sofia 2012 (pages 27-31) [in Bulgarian]

3. Himmel R, Pilo R, Assif D, Aviv I. The cantilever fixed partial denture - - a literature review. $J$ Prosthet Dent. 1992 Apr;67(4):484-7. [PubMed] [CrossRef]

4. Hubalkova H, Linetskiy I. New Trends in Prosthetic Dentistry. Prague Med Rep. 2006; 107(2):149-164.
[PubMed]

5. Maspero C, Farronato D, Giannini L, Farronato G. Orthodontic treatment in elderly patients. Prog Orthod. 2010; 11(1):62-75. [PubMed] [CrossRef]

6. Peev T, Filchev A, Clinic of prosthetic dental medicine. Eko PrintSofia. 2008. [in Bulgarian]

\section{Address for correspondence:}

Dr. Hristina Arnautska, D.M.

Department of Prosthetic Dental Medicine and Orthodontics, Faculty of Dental Medicine - Varna,

150, Tsar Osvoboditel Blvd., Varna, Bulgaria; Tel.: +359 888599678

E-mail: tineia@yahoo.com 